\title{
Traza urbana, uso de suelo industrial y vientos dominantes: confrontación de riesgo en la ciudad de Salamanca, México ${ }^{1}$
}

\author{
Urban layout, industrial land usage and prevailing winds: risk \\ confrontation in the city of Salamanca, Mexico
}

Cómo citar:

Pérez Crespo, J. (2020). Traza urbana, uso de suelo industrial y vientos dominantes: confrontación de riesgo en la ciudad de Salamanca, México. Designia, 8(2), 45-61.

\author{
${ }^{1}$ Investigación con adscripción a la \\ Universidad de Guanajuato, México.
}

* Egresado de la Licenciatura en Arquitectura por el Instituto Tecnológico de Querétaro de la Secretaría de Educación Pública; miembro del Colegio de Arquitectos de Salamanca Asociación Civil; Master en Administración Educativa por la Universidad DelaSalle Bajío; Doctorado en Artes por la Universidad de Guanajuato; Profesor Asociado al Departamento Arte y Empresa de la División de Ingenierías Campus lrapuato-Salamanca, Universidad de Guanajuato; Coordinador del programa educativo en Artes Digitales de la Universidad de Guanajuato (2017-19), Candidato a Investigador del Sistema Nacional de Investigadores del Conacyt. Líneas de investigación: Arte, Arquitectura y Educación. E-mail: armando.perez@ugto.mx ORCID: https://orcid.org/0000-0002-8122-5097

\section{Palabras clave:}

Traza vitruviana, uso de suelo, emisiones industriales, vientos dominantes

Key words:

Vitruvian planning, land use, industrial emissions, prevailing winds

Recibido: $10 / 07 / 2020$

Aceptado: 02/09/2020

Resumen:

El presente artículo participa al lector, de una reflexión que surge a partir de la existencia de la ciudad de Salamanca en el Bajío mexicano, motivada en su origen y crecimiento territorial en los criterios de sanidad implementados en las reales ordenanzas coloniales, y de traza con influencia vitruviana, que no obstante de sus benévolos principios en la configuración de los espacios funcionales y colectivos, en lo evolutivo del asentamiento y su afán de 
progreso, en la segunda mitad del siglo XX se generó una solvencia políticaeconómica nacional, con la necesidad de ocupación o uso de suelo de ámbito industrial, parcialmente resuelto o sin una visión futura ante el comportamiento de las emisiones de los procesos petroquímicos, en los vientos y sus trayectorias, sobre otros usos de suelo principalmente destinados a funciones habitacionales y de servicios, y con latentes consecuencias en la salud de su población; en el presente comunicado se tomaron además, cifras monitoreadas de los compuestos químicos del último año (2019) con valores cuestionables; todo ello, en una confrontación de riesgo entre una traza urbana, un uso de suelo híbrido, y el flujo de emisiones industriales en los vientos del territorio urbano.

\section{Abstract:}

The present article participates the reader to a reflect on the existing city of Salamanca in the Mexican Bajío region. The city's sanitary conditions were implemented during its origins and its later territorial growth under the urban health criteria of the royal colonial ordinances, which were modelled on the methods of Vitruvian planning. Despite its benevolent principles in spatial configuration of the city's functional and collective spaces, and the evolution of the settlement and its desire for progress, the city, in the second half of the 20th century, generated a national political-economic solvency, with a consequent need for occupation or use of industrial land. But this expansion was effected by only partially resolving and without a future vision regarding the behavior of emissions from petrochemical processes in the city refinery. Winds trajectories, over land originally destined for residential services and functions had important consequences for the health of its population; in this present report, statistics obtained from monitoring content of chemical compounds in the last year (2019) along with certain with questionable information were considered; all indicators were used to study the risk confronting the urban layout, and the effects of hybrid land use and flow of industrial emissions, caused by winds flowing across the urban territory under this study. 


\section{EL CONTEXTO.}

La ciudad de Salamanca tiene su fundación en el siglo XVII; actualmente destaca su actividad económica en torno a la paraestatal de Petróleos Mexicanos, cuyo origen de la industria pesada data de la segunda mitad del siglo XX; la sede urbana se precisa en las coordenadas geográficas de $\operatorname{los} 20^{\circ} 34^{\prime} 13^{\prime \prime}$ de latitud norte y los $101^{\circ} 11^{\prime} 50^{\prime \prime}$ de longitud oeste, con una superficie de 774 kilómetros cuadrados a una altura sobre el nivel de mar de 1721 metros (Secretaria del Medio Ambiente y Recursos Naturales SEMARNAT, 2012, pág. 13); Así mismo, su último censo poblacional registra 260,732 habitantes (Instituto Nacional de Estadística y Geografía INEGI, 2015).

La traza vitruviana y su progresiva industrialización.

En todo asentamiento un factor de su configuración territorial es la traza urbana; en el caso de estudio, se podrá referir a los instaurados en las políticas de la Nueva España del año de 1604, en donde las calles rectoras se orientarían a los cuatro puntos cardinales formando una trama reticular, respondiendo a las reales ordenanzas para las nuevas ciudades de la colonia, permitiendo una regulación en su crecimiento y expansión; esta morfología ortogonal también catalogada como renacentista, respondió a los criterios de un aprovechamiento de las cualidades naturales del sitio; de estos criterios fundacionales y del modelo de traza, se menciona:

La selección del sitio para establecer el pueblo nuevo de indios fue guiada por la condición europea que entonces existía para definir qué lugar era sano y conveniente para la vida sedentaria (Kubler, 1984; García Zambrano, 2000). En rigor, las creencias renacentistas con las que contaban los diferentes responsables del proceso de Congregación, no diferían sustancialmente de aquéllas de la Antigüedad basadas tanto en Hipócrates como en Vitruvio (Hipócrates, 1986; Vitruvio. 1997). Era preferible elegir un sitio llano y horizontal que uno irregular sobre pendiente y se aconsejaba contar con un río contiguo al paraje seleccionado en el que se habría de trazar el pueblo. (Fernández Christlieb \& Urquijo Torres, 2006, pág. 149). 
Por lo tanto, en la comprensión morfológica urbana y de sus antecedentes, se asume que desde la antigüedad con los grandes imperios, se tomaron criterios de organización para los espacios públicos; en consecuencia, la disposición de sus lotificaciones responden a trazos geométricos simples, que no únicamente permitieron un control militar sino además, contribuyeron a condiciones de sanidad para sus pobladores, por el flujo y dirección de sus vientos dominantes, como se señala en el concepto de planificación urbana de influencia vitruviana (Vitrubio, 2007) siguiente:

\begin{abstract}
La detección de la cuadrícula romana en nuestras tramas urbanas refleja el carácter práctico y organizador de los antiguos dirigentes del Imperio, que levantaron ciudades simples, claras y fáciles de construir que contribuyeron a la expansión del mismo. También demuestra la esencia de su pensamiento y expone, con su propia geometría, el correcto trazado que tuvo y debería de tener la ciudad. Porque como decía Vitrubio[3] antes de trazar una ciudad había que elegir el lugar adecuado para su emplazamiento, había que hacer un estudio del soleamiento y conocer los vientos, para trazar las calles en dirección opuesta a los mismos y conseguir dispersarlos. Pero sobre todo pone de manifiesto la premisa que dirigió el levantamiento de sus ciudades: el orden como principio jerarquizador de lo urbano. (Tomás Medina, 2017, pág. 15).
\end{abstract}

De ello, el asentamiento salmantino heredó la organización política y territorial para su traza, donde su centro neurálgico recae en la plaza principal; este criterio urbanístico se relaciona con las disposiciones establecidas por el rey aragonés Fernando el Católico en América: "La colonización de las tierras continentales centralizará las funciones administrativas, religiosas, militares y comerciales en ciudades, que se trazan con un arreglo a un entramado regular de calles y manzanas en torno a una plaza central, [...]" (Bielza de Ory, 2002); a partir de esto, al paso de los siglos y en detrimento de lo planificado, se confrontó por vez primera el objetivo de una expansión espacial, contra la necesaria autonomía económica del asentamiento, resolviendo en lo práctico y en la progresiva ocupación de entidades de desarrollo industrial, disponiendo de recursos territoriales sin priorizar en estrategias de planeación 
para ambos hechos; coincidiendo en la postura del urbanista Francisco Sabatini en Conflictos ambientales y desarrollo sustentable de las regiones urbanas (pág. 83), donde describe sobre la presencia de ciertos intereses que de inicio contribuyen al desarrollo económico de una región, pero impactan al no incluir una visión futura en materia de sanidad pública, que por la necesidad de un incremento de equipamientos especializados -industrias-, ya no supone este hecho, una prioridad para la zonificación o planeación de estos, y en consecuencia la aparición de riesgos urbanísticos con afectación en la calidad de vida; de la necesidad de proximidad territorial entre los sectores funcionales donde la sociedad realiza sus actividades vitales (habitacional y laboral); ratificándose en palabras del mismo autor:

\section{[...] la misma planificación urbana puede ser considerada causa de problemas. Primero, la zona funcional homogénea en ciudades de mayor tamaño aleja entre si a gente y actividades que deben interactuar físicamente. Los viajes necesarios crecen en mayor número y extensión, contribuyendo a la congestión, contaminación del aire y demás problemas asociados. Las nuevas tecnologías, catalogadas como "limpias desde el punto de vista ambiental", vuelven posibles e, incluso, recomendables los usos mixtos de suelo como forma de evitar esos viajes. (Sabatini, 1997, pág. 79)}

Considerando los antecedentes de la planeación urbana en México, se ha presentado una débil postura derivada de repercusiones de la industrialización, que en lugar de lograr un diseño sostenible a la par del consecuente crecimiento demográfico, se ha truncado con la orientación de la política con el proceso de industrialización nacional presentado a finales de los años treinta del siglo XX, dejando en otro plano de atención los temas urbanos en favor del pensamiento económico. (Gutiérrez Chaparro, 2009, pág. 60).

\section{Convivencia de emisiones industriales y población, oposición declarada.}

De las actividades encaminadas a la refinación del petróleo en Salamanca y de las necesidades económicas del país, se estimuló a la presencia de industrias petroquímicas que conjunta y conscientemente, han generado emisiones aéreas con riesgo sanitario; el gobierno estatal y con la finalidad de tomar acciones en beneficio de la población ha dispuesto de tres estaciones fijas para el monitoreo de la calidad del aire (Secretaría del Medio Ambiente y Ordenamiento Territorial, 2019), que permiten contar con un registro de emisiones que van desde partículas sólidas con diámetro igual o menor a 10 micrómetros ( $\mu \mathrm{m}$ ) (PM10) a distintos compuestos como el Bióxido o Dióxido de azufre (SO2), el Monóxido de carbono (CO) y otros; esta circulación de 
agentes deriva en factores que afectan la productividad y sanidad en la población (Fedossova, Fedosov, \& Britto, 2019, pág. 334); dicha contaminación forma parte de un sistema desprendido de una organización multivariante en el territorio urbano en ámbitos y funciones entre los equipamientos urbanos con su población; para la ciudad de Salamanca se ha dispuesto de una convivencia velada por décadas de usos de suelo habitacional, con usos industriales del tipo energético, químico, automotriz, y alimentario; resultado de este contexto urbanístico, los habitantes de la zona urbana están expuestos a enfermedades crónicas por el contacto con las emisiones en sus actividades cotidianas, disminuyendo la calidad ambiental del asentamiento:

\footnotetext{
El crecimiento económico y la urbanización, asociados al desarrollo de diversas actividades como la industria petrolera, los servicios, la agroindustria y el incremento de las industrias automotoras, traen como resultado un consumo intenso de combustibles fósiles; al mismo tiempo, la práctica de actividades agropecuarias no apropiadas inciden en la generación de elevados volúmenes de contaminantes, que al relacionarse con las condiciones ambientales pueden dañar la salud humana, los ecosistemas y los recursos materiales. (Romero, Diego, \& Álvarez, 2006, pág. 1).
}

Hoy día en la ciudad de Salamanca, no obstante de la existencia de intensos programas e inversiones en materia de contaminación ambiental dirigidas principalmente a las industrias (Secretaría del Medio Ambiente y Recursos Naturales, 2018), se ha diagnosticado y publicado en el Periódico Oficial del Gobierno del Estado de Guanajuato, una oposición histórica entre la planificación urbana salmantina y la praxis de los usos de suelo para los sectores industriales y restantes, expuesto como sigue: "Debido a las condiciones topográficas y ambientales los sectores de asentamientos humanos e industria no tienen las condiciones más óptimas para desarrollarse en el territorio municipal." (Secretaría de Desarrollo Social y Humano, 2019, 
pág. 150); situación que declara incidentes en el tema sanitario y en las funciones de sus habitantes en diferentes edades, asunto también de competencia global en donde instancias internacionales como la Organización Panamericana de la Salud (OPS), que en su portal web (Pan American Health Organization, 2019) menciona sobre la inequidad de los riesgos y los efectos de la contaminación en la población, señalando en primer lugar como grupos vulnerables a los que cuentan con enfermedades previas; luego al grupo de menores de cinco años y seguido de adultos con edades entre $50 \mathrm{y}$ 75 años; vinculándose estas clasificaciones de edad para Salamanca, en información contenida en las cédulas estadísticas de la Secretaría de Desarrollo Social y Humano estatal, donde la población en mayor número reflejada en la pirámide de edad en 2019, corresponde al rango entre cero y 14 años; en segundo lugar a la población entre los 45 y 64 años; los datos permiten ubicar una proyección poblacional a mediano plazo, en donde al primer grupo de individuos ahora menores, estarán contemplados como población económicamente activa en los próximos 6 años, cuando su primera generación llegue a los 20 años de edad (García Guzmán, 1999); continuando para el segundo grupo de mayoría poblacional y económicamente activa (45-64 años), en donde los de 64 años estarían muy cercanos a un retiro laboral con posibles diagnósticos en salud por atender; ello en un ejercicio de interpretación poblacional, inspirada en la publicación de la investigadora en Los problemas laborales de México a principios del siglo $X X I$ (pág. 14).

\section{Desarrollo económico-industrial e incremento en riesgos sanitarios.}

Con respecto a lo histórico de la economía mexicana y de su industria petrolera, se asume que para el año de 1923, se tienen catorce refinerías en operación, principalmente en la zona costera del Golfo de México, que luego del movimiento político de la expropiación petrolera el 30 de junio de 1950, pone en actividad a la refinería Ingeniero Antonio Manuel Amor (RIAMA) en Salamanca (Vega González \& Rodríguez Román, 2010, pág. 64), complejo industrial habilitado en un sector al noreste de la mancha urbana; con este arranque económico-político se instalarán paulatinamente industrias que hoy día son parte del inventario actuante en el medio ambiente aéreo; de las que entidades iniciales a este uso de suelo industrial, se tiene a la empresa Univex quien inicia sus actividades en los años setenta, con un proceso de cuarenta y dos mil toneladas métricas de crapolactama (crapolactam); de este compuesto la Environmental Protection Agency (EPA) EE.UU. en Acta de Aire Limpio (CAAA) le refiere como un tóxico atmosférico (Anderson \& Patrick, 2006, pág. 340). Otra segunda industria es la denominada Fertilizantes de México (Fertimex) quien dentro de su objetivo de producción inicial considera el satisfacer integral y centralizadamente las necesidades de fertilizantes en el campo de México (Banco Nacional de Comercio Exterior, S.N.C., 1990, pág. 221), de acuerdo con el historial 
de la Fertimex-Mostrose, nace en 1943 con actividades de explotación, producción y venta de guanos y fertilizantes, generando contaminantes con compuestos clorados, fosforados y otros; en 1968 produce insecticidas organofosforados (metil, paratión y malatión); entre noviembre de 1992 y febrero de 1993 la Procuraduría Federal de Protección al Medio Ambiente (PROFEPA) le realizó una auditoría ambiental, en estos años llamada Tekchem, detectando la acumulación de residuos peligrosos generados por 35 años de operación (Secretaría del Medio Ambiente y Recursos Naturales, 2018, págs. 11-13); sumándose, que el 12 de septiembre del año 2000 un accidente provocó una fuga de toneladas de insecticida malatión, precipitándose en forma de lluvia sobre la ciudad (Arreola, 2000). Otra paraestatal en el mismo sector y de relevancia en la región centro-norte de México, es la Comisión Federal de Electricidad (CFE), creada en el año de 1937 y nacionalizada en 1960; en el estudio Del discurso a la aplicación práctica en materia de energía y en sus capítulos de Hacía la sustentabilidad ambiental de la producción de energía en México, (Seefoó Lujan , 2014, pág. 347) se declara sobre las industrias generadoras de electricidad, exponiendo que para México, las emisiones de Gases de Efecto Invernadero (GEI) representan un reto para su disminución, ya que los insumos para la generación de electricidad se sustentan fuertemente en hidrocarburos como el gas natural, el combustóleo y el diesel hasta en un $67.1 \%$ (2008), y que no pareciera disminuir por el incremento de los factores análogos de la urbanización y la presencia industrial; en su sexto capítulo se menciona a la generación eléctrica y las externalidades ambientales como un impacto subsecuente en la salud, con el ejemplo para la zona metropolitana de la ciudad de Monterrey, cuyas emisiones de partículas finas (PM2.5) surgen por reacciones atmosféricas de los contaminantes primarios como el dióxido de azufre (SO2) y los óxidos de nitrógeno (NOX) (Cita de Macias Guzmán, 2011 en Seefoó); este tipo de industria energética, al estar ubicada en un radio de 700 kilómetros hacia el centro de población, resultaría en asociaciones a casos de mortalidad y morbilidad 
cardiopulmonar; así como esta ciudad, otros asentamientos forman parte de este grupo en condiciones ambientales críticas: "[...] tomando en cuenta a seis fuentes emisoras operadas por CFE a saber: Río Escondido, Carbón II (Coahuila), Altamira, Tuxpan (Veracruz), Villa de Reyes (San Luis Potosí) y Salamanca (Guanajuato)." de igual manera Macias Guzmán (pág. 348) enfatiza la ausencia de estudios paralelos que consideren variables a influir en la contaminación como la dirección del viento, diferencias entre la población urbana y rural, y el uso del suelo.

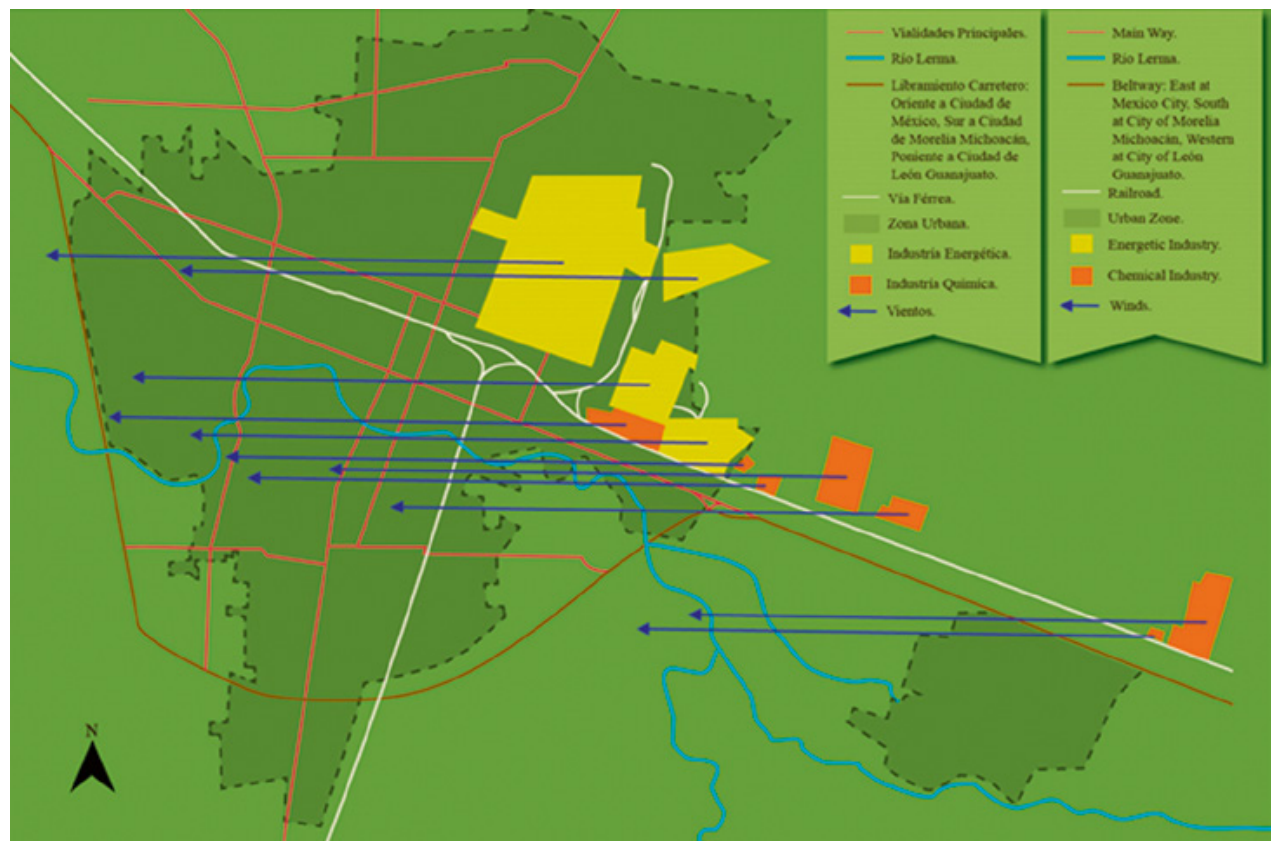

Gráfico 1. Zona urbana de la ciudad; al noreste la ubicación del uso de suelo industrial; las fechas en azul señalan la dirección histórica de los vientos dominantes del noreste, cuyas emisiones cruzan los usos de suelo restantes (habitacional y de servicios). 


\section{Emisiones industriales y vientos dominantes.}

Del inventario mencionado, se identificó al noreste de la ciudad una gran presencia por más medio siglo de industrias del sector químico-energético; por la ubicación adjunta a los restantes usos de suelo: centro histórico y habitacional principalmente; surge entonces el cuestionamiento sobre el comportamiento entre la operatividad industrial, el uso de suelo y la dirección de los vientos dominantes sobre la traza urbana; de acuerdo con los reportes de la Secretaría de Medio Ambiente y Recursos Naturales (SEMARNAT) y del Gobierno del Estado de Guanajuato (Instituto Nacional de Ecología y Cambio Climático. Secretaría del Medio Ambiente y Recursos Naturales, 2016, pág. 8) en su informe de Descripción climatológica de la región para el municipio de Salamanca señala: "Con base a la información de la red de monitoreo atmosférico, históricamente se ha observado que la región de Salamanca presenta vientos dominantes del Noreste con una mayor frecuencia de vientos de 2 a $4 \mathrm{~m} / \mathrm{s}$, seguido de vientos de 4 a $7 \mathrm{~m} / \mathrm{s}$.", y de acuerdo con la existencia de tres estaciones de monitoreo en la ciudad, en un polígono con una distancia de dos kilómetros entre si, se procedió a extraer las cifras más representativas (2019) anotadas en la Tabla I, en la primera columna de la izquierda se tienen los compuestos químicos de emisión, seguido de los meses en que los valores de emisión resultaron críticos, meses en que la dirección del viento proviene del noreste (zona industrial) al suroeste sobre los restantes usos de suelo; en las columnas 3 y 4 aparecen los valores máximos monitoreados y los permisibles por las Normas Oficiales Mexicanas (NOM) respectivamente, cifras contrastantes (Sistema Nacional de Información de la Calidad del Aire, SINAICA, 2020, pág. 24) que pueden resultar en una alta afectación en los grupos más sensibles de la población -niños y adultos mayores-, en padecimientos respiratorios y cardiovasculares (Secretaría del Medio Ambiente y Ordenamiento Territorial , 2020, pág. 74). 


\begin{tabular}{|c|c|c|c|}
\hline 1. Tipo de emisión & $\begin{array}{c}\text { 2. Mes de registro } \\
\text { crítico de la } \\
\text { emisión en 2019 }\end{array}$ & $\begin{array}{c}\text { 3. Valor máximo } \\
\text { registrado de la } \\
\text { emisión }\end{array}$ & $\begin{array}{c}\text { 4. Valor NOM } \\
\text { permisible de } \\
\text { emisiones }\end{array}$ \\
\hline Dióxido de Azufre (SO2) & Febrero & $0.13757 \mathrm{ppm}$ & $0.08 \mathrm{ppm}$ \\
\hline Monóxido de Carbono (CO) & Febrero & $0.14635 \mathrm{ppm}$ & $0.13 \mathrm{ppm}$ \\
\hline Dióxido de Nitrógeno (NO2) & Febrero & $6.94 \mathrm{ppm}$ & $11 \mathrm{ppm}$ \\
\hline PM10 & Abril & $491.12 \mu \mathrm{pg} / \mathrm{m} 3$ & $120 \mu \mathrm{m} / \mathrm{m} 3$ \\
\hline PM2.5 & Mayo & $154.37 \mu \mathrm{g} / \mathrm{m} 3$ & $65 \mu \mathrm{m} / \mathrm{m} 3$ \\
\hline $\begin{array}{l}\text { Descripción de las unidades de concentración: } \\
\text { ppm = partículas por millón. } \\
\mu \mathrm{g} / \mathrm{m} 3=\text { microgramo por metro cúbico. }\end{array}$ \\
\hline
\end{tabular}

Tabla 1. Descripción

Fuente: autor.

Sobre los compuestos químicos referidos, en la publicación Efectos de la contaminación atmosférica en la salud y la importancia en la ciudad de México (Vallejo, Jáuregui-Renaud, Hermosillo, Márquez, \& Cardénas, 2003) se describe al ozono como un constituyente negativo que recae en las vías respiratorias con síntomas reflejados en tos, sibilancias, cefalea, náusea e irritación conjutival de nariz y faringe (p. 58); por otra parte, el dióxido de azufre generado por la ignición de combustibles fósiles tiene un precedente de la Ministry of Heafth (1954) citado también por Vallejo (pág. 59):

En las áreas urbanas cuando la contaminación por bióxido de azufre se asocia a contaminación por bióxido de nitrógeno y monóxido de carbono puede tener consecuencias fatales. En Londres en 1952, el complejo de óxido de azufre y partículas ("la niebla negra") fue el responsable de un aumento de la mortalidad especialmente de personas de enfermedad cardiaca o respiratoria y en niños pequeños. 
De forma similar el monóxido de carbono, en una concentración mayor al 4.2\% de carbioxihemoglobina altera la percepción, las funciones cerebrales y la disminución de reflejos osteomusculares hasta provocar la muerte en caso extremo. En lo que se refiere a las partículas suspendidas del rango PM10 y PM2.5 superadas en los rangos normativos de la Tabla I; se tiene que su exposición en concentraciones altas por breves periodos en población de adultos, niños, pacientes con enfermedades cardiorrespiratorias y con asma, se les relacionará con el aumento en la morbilidad por enfermedad pulmonar obstructiva crónica y neumonía; y en la mortalidad por cardiopatía isquémica.

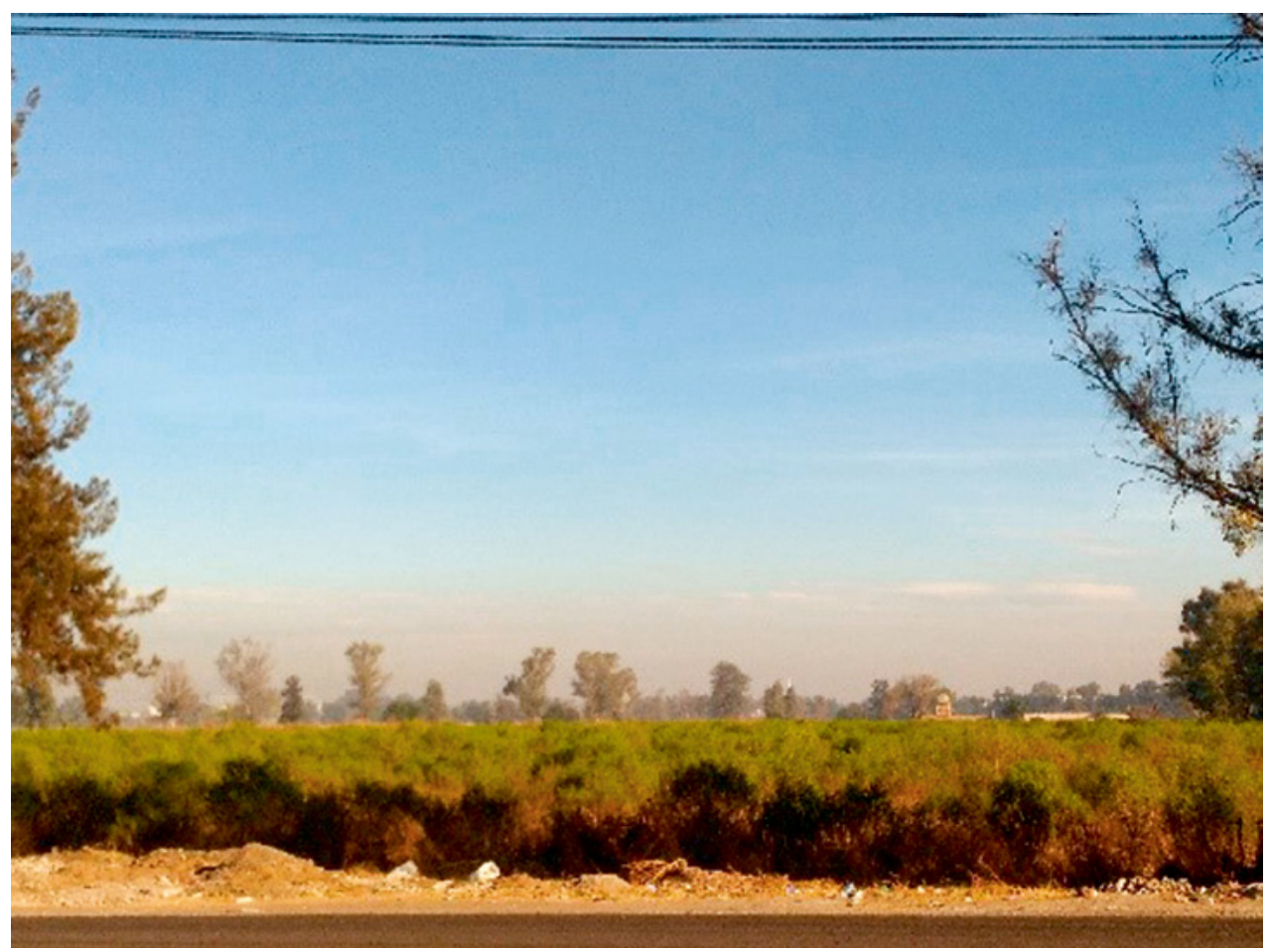

Figura 1. Vista en el perímetro suroeste de la mancha urbana, opuesta a la zona industrial; nótese sobre el horizonte el denso estrato correspondiente a su contaminación. 


\section{CONCLUSIONES}

Hoy día el uso de suelo ocupado por la industria en Salamanca se estima en un veinte por ciento; si bien, en la fundación del asentamiento se dispuso de una benévola traza ortogonal para sus calles, influida en los principios vitruvianos para mitigar alguna amenaza sanitaria sobre su población; en lo subsecuente, la ciudad fue partícipe de un plan de desarrollo nacional en los rubros económico-industrial desde la segunda mitad de siglo $X X$, teniendo hasta ese momento, usos de suelo compatibles pero no formales, entre lo habitacional, comercio, industrias ligeras y de almacenaje, con un perímetro territorial con vocación al uso en la agricultura e inclusive en la pesca; más sin embargo, el progreso suscitado en las ciudades ha sido un aspecto vulnerable en la planeación urbana en México, que antepone la necesidad de satisfacer una economía sustentable al Estado y en segundo término una consideración a su población, sin un contundente ejercicio en materia medioambiental y de usos de suelo a largo plazo; por otra parte, se ha declarado para la ciudad, la no optimización entre las condiciones topográficas y ambientales con los asentamientos humanos (Secretaría de Desarrollo Social y Humano); y también en lo histórico del tema de los vientos dominantes desplazados desde el cuadrante industrial noreste (Instituto Nacional de Ecología y Cambio Climático), vientos con emisiones que transportan compuestos químicos fuera de normativa, con una alta probabilidad en efectos de mortalidad y morbilidad cardiopulmonar para la población, equiparable al de las grandes urbes en condiciones críticas. ¿Qué proponer entonces? Una tendencia sería el crecimiento urbano periférico; partiendo del hecho de que el centro histórico y los usos de suelo complementarios, más la densidad poblacional, demandarán una constante actualización y mejora en la 
infraestructura por sus circunstancias de antigüedad y saturación; por lo tanto, será necesario liberar los usos de suelo conflictuados ampliándoles territorialmente con calidad urbanística en sus espacios colectivos y de equipamientos; un nuevo plan maestro con usos de suelo que contengan espacios suburbanos periféricos con funcionalidades especificas sustentables: gobierno, comercio especializado, parques tecnológicos e industriales, zonas habitacionales entre otras; sobre el tema de las industrias sin emisiones de riesgo, Sabatini ya mencionaba el uso de energías limpias para su operación, alusivo a ello y al concluir la presente investigación se publicó favorablemente para la sociedad, una resolución de controversia entre la Secretaría de Energía (SENER) Federal y Greenpeace México, ante la negativa del uso de energía renovable, así como de pruebas preoperativas en las centrales eólicas y fotovoltaicas, derivado de acuerdos internacionales en materia de cambio climático (Salgado, 2020). Finalmente, se esperaría una suma de voluntades entre todos los sectores e integrantes del asentamiento humano, para una armoniosa relación entre el clima, la salud en la población económicamente activa presente y futura; y el equipamiento industrial con emisiones limpias. 


\section{REFERENCIAS BIBLIOGRÁFICAS}

Anderson, E. L. \& Patrick, D. R. (2006). Medio ambiente en desarrollo. Control de la contaminación atmosférica en EE. UU. Providencia de Santiago de Chile, Chile: Centro de Estudios Püblicos CEP. Obtenido de https:// www.cepchile.cl/cep/site/artic/20160304/asocfile/20160304093942/16_cap4anderson_libromambiente.pdf

Arreola, J. J. (2000 de Octubre de 2000). Solicitan informe tras fuga de Malation. (E. Universal, Ed.) Recuperado el 2019, de https://archivo.eluniversal.com.mx/estados/17336.html

Banco Nacional de Comercio Exterior, S.N.C. (1990). Aspectos de la industria de fertilizantes. comercio exterior, 3(40), 221-226. Recuperado el 2019, de http://revistas.bancomext.gob.mx/rce/magazines/160/12/ CE_MARZO_1990.pdf

Bielza de Ory, V. (15 de Enero de 2002). De la ciudad ortogonal aragonesa a la cuadricular hispanoamericana como proceso de innovación-difusión, condicionado por la utopía. (U. d. Barcelona, Ed.) Revista Electrónica de Geografía y Ciencias Sociales, VI(106). Recuperado el 2019, de http://www.ub.edu/geocrit/sn/sn-106.htm

Fedossova, A., Fedosov, V. V. \& Britto, R. A. (2019). Dispersión de las partículas de emisiones industriales en el problema de la optimización semi-infinita del área contaminada. Información Tecnológica, 30(1), 333-344. doi:http://dx.doi.org/10.4067/S0718-07642019000100333

Fernández Christlieb, F. \& Urquijo Torres, P. S. (2006). The spaces of the pueblo de indios after the process of Congregación, 1550-1625. (B. d. Geografía, Ed.) Investigaciones geográficas(60), 145-158. Recuperado el 2019, de http://www.scielo.org.mx/scielo.php?pid=S0188-46112006000200009\&script=sci_arttext

García Guzmán, B. (1999). Los problemas laborales de México a principios del siglo XXI. Papeles de Población, 5(21), 9-19. Recuperado el 2019, de https://www.redalyc.org/pdf/112/11202102.pdf

Gutiérrez Chaparro, J. J. (2009). Planeación urbana en México: Un análisis crítico sobre su proceso de evolución. Urbano, 12(19), 47-63. Recuperado el 2019, de http://revistas.ubiobio.cl/index.php/RU/article/view/335

Instituto Nacional de Ecología y Cambio Climático. Secretaría del Medio Ambiente y Recursos Naturales. (2016). Evolución y comportamiento de las concentraciones de contaminantes atmosféricos en un sitio en Salamanca Guanajuato. Coordinación General de Contaminación y Salud Ambiental. Recuperado el 2019, de https://www.gob.mx/cms/uploads/attachment/file/250911/ReporteSalamancaAO_lab_movil.pdf 
Instituto Nacional de Estadística y Geografía INEGI. (2015). Salamanca Guanajuato (11027). Recuperado el 2019, de https://www.inegi.org.mx/app/indicadores/?t=0200\&ag=070000110027

Pan American Health Organization. (2019). Organización Panamericana de la Salud. Recuperado el 2019, de https://www.paho.org/hq/index.php?option=com_content\&view=article\&id=12918:ambient-air-pollution\&Itemid=72243\&lang=es

Romero, M., Diego, F., \& Álvarez, M. (2006). La contaminación del aire: su repercusión como problema de salud. Revista Cubana de Higiene y Epidemiología, 44(2), 1-14. Recuperado el 2019, de http://scielo. sld.cu/pdf/hie/v44n2/hie08206.pdf

Sabatini, F. (1997). Conflictos ambientales y desarrollo sustentable de las regiones urbanas. Revista EURE - Revista de Estudios Urbano Regionales, 23(68), 77-91. Recuperado el 2019, de http://eure.cl/index. php/eure/article/view/1157

Salgado, A. (Mayo de 2020). (C. Magazine, Productor, \& Themebeez) Recuperado el 2020, de https:// aliciasalgado.mx/2020/05/juez-federal-suspende-la-politica-energetica-de-la-sener/

Secretaría de Desarrollo Social y Humano. (2019). Programa Municipal de Desarrollo Urbano y Ordenamiento Ecológico Territorial de Salamanca, Abreviada. (G. d. Guanajuato, Editor) Recuperado el 2019, de https:// portalsocial.guanajuato.gob.mx/sites/default/files/documentos/2016_SALAMANCA_Programa_ desarrollo_urbano_ordenamiento_ecologico_territorial_1.pdf

Secretaría del Medio Ambiente y Ordenamiento Territorial . (2020). Programa de gestión para mejorar la calidad del aire de Salamanca, Celaya e Irapuato 2013-2022. Recuperado el 2019, de https://smaot.guanajuato. gob.mx/sitio/calidad-del-aire/7/PROAIRE-Salamanca

Secretaría del Medio Ambiente y Ordenamiento Territorial. (2019). SEICA Monitoreo Atmosférico Salamanca. Recuperado el 2019, de https://smaot.guanajuato.gob.mx/sitio/seica/monitoreo/salamanca 
Secretaría del Medio Ambiente y Recursos Naturales. (2018). Libro Blanco Acciones y obras realizadas y en proceso, para el manejo ambientalmente adecuado de residuos y remediación del sitio contaminado en la ex unidad industrial de Fertimex-Tekchem, Salamanca Guanajuato. Recuperado el 2019, de http://dsiappsdev. semarnat.gob.mx/datos/portal/transparencia/2018/Libro_Blanco_Fertimex.pdf

Secretaría del Medio Ambiente y Recursos Naturales. (31 de octubre de 2018). Plan Salamanca. Recuperado el 2019, de https:/www.gob.mx/semarnat/acciones-y-programas/plan-salamanca

Secretaria del Medio Ambiente y Recursos Naturales SEMARNAT. (2012). Programa para mejorar la Calidad del Aire en Salamanca 2007-2012. Dirección de la Calidad del Aire, Dirección General de Gestión de la Calidad del Aire y Registro de Emisiones y Transferencia de Contaminantes. SEMARNAT. Recuperado el 2019, de https://www.gob.mx/cms/uploads/attachment/file/311633/Informe_ProAire_ Salamanca_E11.pdf

Seefoó Lujan , J. L. (2014). María Eugenia González Ávila, Gabriela Muñoz Melendez y Alfredo Ortega Rubio, coords. Hacía la sustentabilidad ambiental de la producción de energía en México. El Colegio de la Frontera Norte, Centro de Investigaciones Biológicas del Noroeste 2011, 268 p. Relaciones. Estudios de historia y sociedad, 35(139), 343-350. Recuperado el 2019, de http://www.scielo.org.mx/ scielo.php?script=sci_arttext\&pid=S0185-39292014000300015

Sistema Nacional de Información de la Calidad del Aire, SINAICA. (2020). Manual 1 Principios de la Medición de la Calidad del Aire. Recuperado el 2019, de https://sinaica.inecc.gob.mx/archivo/guias/1-\%20 Principios\%20de\%20Medici\%C3\%B3n\%20de\%20la\%20Calidad\%20del\%20Aire.pdf

Tomás Medina , C. (2017). La historia urbana como entedimiento de fundamental para el entendimiento de la ciudad. Jornadas Internacionales sobre los nuevos crecimientos urbanos (10. 2017. Sevilla) (págs. 13-22). Sevilla, España: Deposito de Investigación Universidad de Sevilla idUS. Recuperado el 2019, de https://hdl.handle.net/11441/81614

Vallejo, M., Jáuregui-Renaud, K., Hermosillo, A. G., Márquez, M. F., \& Cardénas , M. (2003). Efectos de la contaminación atmosférica en la salud y su importancia en la ciudad de México. Gaceta Médica de México, 1(139), 57-63. Recuperado el 2019, de https://www.medigraphic.com/cgi-bin/new/resumen. cgi?IDARTICULO=1108

Vega González , L. R., \& Rodríguez Román, L. A. (2010). Metodología para determinar el tiempo de respuesta de las carreras de ingeniería a las necesidades operativas de la plataforma tecnológica de la industria mexicana. Revista Universidad EAFIT, 46(157), 55-74. Recuperado el 2019, de https:// www.redalyc.org/pdf/215/21520964005.pdf

Vitrubio, M. L. (2007). Los diez libros de arquitectura. (A. Blánquez, Trad.) Barcelona: Iberia. Obtenido de https://idus.us.es/handle/11441/81614 